Boletín de la Sociedad Geológica Mexicana

VOLUMEN 62, NÚM. 1, 2010, P. 109-122

\title{
Cooperación en el campo de la pequeña minería en Sudamérica: El papel de las ONGs
}

\author{
Claudia Mesa ${ }^{1, *}$, Pura Alfonso ${ }^{1}$, Eva Monterde ${ }^{1}$, Marc Costa $^{1}$ \\ ${ }^{1}$ Departament d'Enginyeria Minera i recursos Naturals, Universitat Politècnica de Catalunya. Av. de les Bases de Manresa, 61-73 \\ Manresa, 08242. España. \\ *cmesa@gencat.cat
}

\section{Resumen}

A lo largo de la historia de la cooperación la trayectoria de las ONGs ha ido evolucionando y el papel vertical de las organizaciones asistencialistas del siglo pasado ha dado paso a una nueva generación de ONGs que trabajan para y con las comunidades con las que cooperan. En la actualidad, un proyecto, desde su nacimiento hasta su ejecución, tiene que formar parte de la comunidad en la que se trabaja.

De todos los campos de actuación de la cooperación internacional en Sudamérica, la minería es uno de los sectores en el que menos se invierte, ya sea por su desconocimiento como por la imagen negativa que de ella posee parte de la población, especialmente en países desarrollados. Sin embargo, las ONGs técnicas pueden colaborar con la pequeña minería según las necesidades requeridas en cada una de las fases del ciclo mismo de la exploración y explotación minera: fases de exploración e investigación, el cálculo de reservas según la ley de mercado, el óptimo diseño de la explotación, que minimice riesgos, maximice ganancias y sea más tolerable con el entorno, el diseño de un correcto plan de restauración y los correspondientes estudios de impacto ambiental. Estos proyectos se desarrollan en el seno de programas que engloban aspectos técnicos, de productividad, sociales y culturales, de género y trabajo infantil, comunitarios, organizativos, ambientales, de salud y seguridad, legales, de formalización, laborales, económicos, de financiamiento y de gestión empresarial, que tienen como objetivo principal la mejora en la calidad de vida de todas aquellas personas vinculadas con el trabajo en la pequeña minería y la minería artesanal y el desarrollo de sus comunidades mineras.

Palabras clave: Sudamérica, minería artesanal, cooperación, desarrollo, ONG.

\begin{abstract}
The trajectory of NGOs has been evolving throughout their history of cooperation and during the last century the "vertical aid" programs of these organizations have given way to a new generation of NGOs that work for and with the communities with which they cooperate. Nowadays, a project, from its birth up to its execution, has to be part of the community.

The mining industry is one of the sectors that has the least amount of foreign investment in South America among all the fields of activity of international cooperation, either because of a lack of knowledge on this subject or because of the negative image held by many people on mining, particularly in the developed countries. Nevertheless, the technical NGOs can collaborate with the small mining industry according to the needs required in each phase of the cycle of mining exploration and exploitation: exploration and research, calculation of reserves according to free market laws, the ideal design of exploitation, which minimizes risks, maximizes earnings and causes as little harm possible to the environment, the design of an acceptable land restoration plan and the corresponding environmental impact studies. These projects develop as part of programs that include different aspects: technical, productivity, social and cultural, gender, child labor, community, organizational, environmental, health and safety, legal, formalization, labor practices, economic, financing and business management. The main objective of these programs is the improvement in quality of life of all the people linked with the work in the small-scale and artisanal mining industry and the development of their mining communities.
\end{abstract}


Keywords: South America, artisanal mining, cooperation, development, NGO.

\section{Introducción}

A lo largo de la historia de la cooperación la trayectoria de las ONGs ha ido evolucionando en su concepción y el papel vertical de las ONGs asistencialistas del siglo pasado, cuya máxima era ir a ayudar transitoriamente a una determinada comunidad del sur, ha dado paso a una nueva generación de ONGs que trabajan para y con las comunidades con las que cooperan. En la actualidad, las ONGs no buscan a sus socios para ejecutar un proyecto concebido desde el norte, sino que se pretende que sea el socio del sur el que comunique sus necesidades a la ONG y ésta, en cooperación con el beneficiario, siente las bases para la realización de un proyecto que desde su nacimiento hasta su ejecución deberá sentirse propio de la comunidad en la que se trabaja. La finalidad de este procedimiento es que cuando una vez finalizados los proyectos de cooperación el socio del norte desaparezca, la contraparte del sur sea lo suficientemente autónoma y sienta el proyecto tan suyo que quiera y pueda seguir ejecutándolo sin ningún problema.

Las ONGs de carácter técnico trabajan para el desarrollo de proyectos que se desenvuelven dentro de un Enfoque de Marco Lógico (en adelante EML), en el que se realiza una evaluación continua sobre los resultados esperados y los obtenidos, del mismo modo que se aboga por la calidad y los resultados y no por la pura satisfacción personal del buen samaritano como podía pasar en otros tiempos.

En todas las fases del desarrollo de un proyecto pueden existir particularidades que hagan que éste fracase, pero es en su identificación cuando corre más riesgos, pues un mal análisis de la situación actual y de la problemática puede provocar que se dediquen muchos esfuerzos a un proyecto condenado al fracaso desde su concepción.

Es por ello que para que los proyectos de cooperación sean fructuosos, las ONGs que los realizan tienen que tener claros cuáles son sus ámbitos de actuación y así saber si son capaces de solventar la problemática que se les plantea o mejor dejar esas actuaciones para otras ONGs.

Las ONGs que desarrollan su actividad en el campo de la minería centran muchos de sus esfuerzos en el campo de la pequeña minería, ya que (1) es ésta la que presenta con mayor intensidad deficiencias en sus diferentes fases de actuación y (2) este tipo de minería produce unas ganancias mejor distribuidas entre la población con un beneficio social mucho mayor (Noetstaller, 1994).

Si dentro de la pequeña minería englobamos a la minería artesanal encontraremos cuadrillas de trabajo basadas en grupos familiares que no poseen la formación técnica adecuada para desarrollar un trabajo sin riesgos para ellos y el entorno (ej. Butt, 1981), y mucho menos conocimientos de la geología de los depósitos que pretenden explotar. Estos grupos de mineros, que en la mayoría de ocasiones operan al margen de las leyes de los países en los que se sitúan, trabajan, en muchos casos, con métodos que no han variado desde la época colonial y que les permiten llevar una vida cuando menos humilde, además de generar un impacto ecológico considerable (Spiegel y Veiga, 2005).

Llegado este punto, cabe preguntarse qué puede hacer una ONG para ayudar a mejorar la calidad de vida de los habitantes de regiones mineras donde se practica la minería artesanal, en qué fases del planteamiento minero pueden cooperar las ONGs y cómo se deben plantear estos proyectos de cooperación.

\section{Situación actual en América Latina}

América Latina posee una gran riqueza en cuanto a recursos humanos y naturales, hecho que le ha proporcionado un aumento económico en los últimos años, pero también es una de las regiones de la Tierra donde la desigual repartición de las riquezas ha desembocado en un elevado nivel de pobreza. La consecución de los Objetivos de Desarrollo de Milenio propuestos por la ONU implicaría la modificación del modelo de desigualdad a favor del crecimiento global, la transmisión equitativa de servicios sociales y el ofrecimiento de oportunidades económicas para las personas más pobres; pero si no se producen cambios drásticos, en breve la región no alcanzará los objetivos previstos para el 2015 y el progreso seguirá siendo desigual. Para lograr una disminución de la pobreza en Latinoamérica se tienen que mejorar el comercio y la integración, reforzar la competitividad, aprender a explotar sus recursos naturales e identificar nuevas oportunidades entre los mercados mundiales, además de invertir en la investigación y el desarrollo y mejorar la producción gracias al uso de nuevas tecnologías (UNPD, 2007).

Ante el contexto de desigualdad y pobreza que se vive alrededor de todo el planeta y, en especial, en regiones como América Latina, se han creado diferentes organizaciones, asociaciones y grupos de distinta índole que, con distintas finalidades y objetivos, se han lanzado a la realización de campañas de financiamiento y/o trabajo con la intención de mejorar la situación global en las zonas en las que se requiera.

En la Declaración del Milenio, realizada por la ONU (2000, Tabla 5) se recogen ocho objetivos de desarrollo para mejorar la situación actual de gran parte de la humanidad, referentes a la erradicación de la pobreza, la educación primaria universal, la igualdad entre los géneros, la mortalidad infantil y materna, el avance del VIH/SIDA y la sostenibilidad del medio ambiente. El octavo objetivo 
Tabla 5. Objetivos de desarrollo de la declaración del Milenio realizado por la ONU en 2000 (ONU, 2000).

I Erradicar la pobreza extrema y el hambre

II Lograr la educación universal

III

Promover la igualdad de género y autonomía de la mujer

IV Reducir la mortalidad infantil

V Mejorar la salud materna

VI Combatir el VIH/SIDA, Malaria y otras enfermedades

VII Garantizar la sostenibilidad del medio ambiente

VIII Fomentar una asociación mundial para el desarrollo

promueve que el sistema comercial, de ayuda oficial y de préstamo garantice la consecución en el 2015 de los primeros siete objetivos fomentando un mundo más justo. Por otro lado, estos objetivos se han mirado desde un punto de vista crítico ya que, aunque cargados de buena voluntad, son demasiado generalistas y poco realistas para conseguir las metas propuestas para el año 2015, según los parámetros de evaluación que van siendo examinados por un grupo de expertos (ONU, 2008).

Numerosas organizaciones dedicadas a la cooperación para el desarrollo, intencionadamente o no, impulsan de una manera u otra uno o más de estos objetivos con sus planes de actuación. Asimismo, las diferentes ONGs y agencias de cooperación que trabajan para mejorar la situación de las personas relacionadas con la pequeña minería y la minería artesanal enfocan sus trabajos para conseguir, en diferentes grados y modos, más de uno de los objetivos y, principalmente, la reducción de la pobreza extrema y el hambre (Hruschka, 2002). A partir de la obtención de este objetivo principal, los trabajos de cooperación también se centran en otros fines, como son promover la igualdad de género y garantizar la sostenibilidad del medio ambiente. Cabe entender que los proyectos de cooperación, además de ser en muchos casos multidisciplinares, contemplan todo el conjunto de características de una localidad (sociológicas, culturales, económicas, tecnológicas, ambientales, etc.) para poder mejorar la situación en la que se encuentra. Cabe recordar que gran parte de los proyectos de cooperación para el desarrollo están englobados en programas que realizan muchísimas actuaciones de distinta índole para poder lograr los objetivos específicos propuestos.

La Organización Internacional del trabajo (OIT, 1999) estimó que la minería artesanal involucró a alrededor de 13 millones de personas en 1998 en 55 países y sugiere que entre 80 y 100 millones de personas en todo el mundo dependen directa o indirectamente de esta actividad. Actualmente se considera que estas cifras se han incrementado, especialmente en la minería del oro (Veiga et al., 2006).

En la actualidad gran parte de la población de muchos países latinoamericanos vive, o sobrevive, a partir de los beneficios que le aporta la pequeña minería. Poniendo una lupa sobre Ecuador, uno de los países en los que la ONG Minería Para el Desarrollo (MPD) ha cooperado, se estima que en el año 2000 había un total de 92,000 personas que trabajaban directamente en la minería a pequeña escala, de las cuales 60,000 trabajaban en la minería metálica y 32,000 en la no metálica; de este total de personas 81,200 son hombres, 6,200 mujeres y 4,600 niños. Asimismo, existen unas 25,000 personas involucradas de forma indirecta en la pequeña minería ecuatoriana, desarrollando actividades como la comercialización de provisiones y enseres para las familias mineras, trabajando en la construcción de edificaciones industriales y viviendas y laborando en los diferentes talleres relacionados con la industria minera. De las ganancias derivadas de las actividades relacionadas con la minería aurífera a pequeña escala, el $80 \%$ de los ingresos se invierten directamente en el país, mientras que el restante $20 \%$ se destinaba a la adquisición de nueva maquinaria, repuestos e insumos del mercado internacional. Otro efecto de la minería es el nacimiento de pueblos (El Oro, Gena, Naranjillas, etc.), la construcción de nuevas vías de comunicación y la activación del transporte automotor (Sandoval, 2001). De todo esto se extrae que en la actualidad la pequeña minería, lejos de ser una actividad residual y en decadencia, es una fuente de ingresos para una buena parte de la población y uno de los motores de la economía del país.

Por otro lado, como se ha dicho anteriormente, la minería, y en especial la pequeña minería y la minería artesanal, puede tener unos graves efectos negativos sobre las personas que trabajan en ella (trabajo infantil, poca seguridad y elevados riesgos laborales, desconocimiento de las técnicas adecuadas, no accesibilidad a las últimas tecnologías, etc.) y sobre el medio en el que se ejecutan las labores. Los efectos negativos de la minería sobre el medio ambiente y la salud se ven acuciados en la minería metálica y especialmente en la minería del oro, que es una de las más extendidas en países latinoamericanos. Siguiendo con el ejemplo de Ecuador, se ha observado que la minería a pequeña escala genera un cambio en los usos del suelo, produciendo una pérdida de tierras agrícolas y habitables (Zaruma y Portovelo), una disminución de la biodiversidad, la incorporación de metales pesados en diferentes organismos y acumulación de mercurio en los mismos (Río Puyango, Río Siete y Ríos Gala y Chico), una pérdida en agua potable para la irrigación y para el cultivo acuático (Río Siete) e impactos en la salud humana. Asimismo, se debe mencionar que la actividad minera a nivel nacional es artesanal en un $82 \%$, que el $97 \%$ de los trabajos se realiza sin ningún tipo de planificación técnica y que el $99 \%$ de las canteras no cumplen con las normas del Instituto Ecuatoriano de Normalización agravando la situación de la pequeña minería en general (Sandoval, 2001).

Otros ejemplos de minería a pequeña escala los tenemos en Bolivia donde ésta aporta el 35\% de la producción nacional de minerales y se localiza en las zonas de Oruro, 
Potosí y La Paz (Ekamolle, 2002). Los asentamientos mineros carecen de servicios básicos como agua potable, electricidad, servicios de salud e infraestructura educativa adecuada. Existen problemas de consumo de alcohol, además de carencias nutricionales y de salubridad, además, el trabajo infantil es una problemática constante. La minería artesanal usualmente se encuentra en el umbral de la informalidad o la ilegalidad al carecer de títulos mineros o licencias ambientales.

En Brasil, el 73\% de los Garimpeiros (mineros artesanales o de pequeña escala) se dedican a la producción del oro y se concentran primordialmente en la Amazonía. El Garimpo constituye una de las principales fuentes de empleo en minería con un alto índice de informalidad y constantes problemas de salud y seguridad.

En Chile la minería en pequeña escala se concentra principalmente en las regiones de Atacama y Coquimbo, donde los trabajadores viven con sus familias y es usual que sus padres y parientes hayan trabajado en la misma actividad.

En Perú los programas e iniciativas de apoyo a la minería a pequeña escala (MPE) se ejecutan fundamentalmente a través de la ENAMI (Empresa Nacional de Minería), que compra el mineral que producen los mineros en pequeña escala y lo procesa en las plantas de beneficio que posee (Ekamolle, 2002).

Estos datos, muy similares en toda Latinoamérica, en menor o mayor grado, son más que suficientes para que una organización que se dedica a la cooperación en el marco de la minería dirija gran parte, sino todos sus proyectos, para la mejora de la situación de las comunidades mineras, en especial aquellas dedicadas a la pequeña minería y en concreto a la minería de tipo artesanal, que es la menos tecnificada, la menos asesorada y la más peligrosa para los trabajadores y el medio ambiente.

Tratándose la pequeña minería de una actividad de alto riesgo en la que trabaja un gran número de personas, entre ellas niños/as, y que genera una gran riqueza en el país de origen, se puede llegar a pensar que mejorando sus condiciones actuales se ayudaría a conseguir el Objetivo Para el Desarrollo número uno de la ONU que consiste en erradicar la pobreza extrema.

Debido a la gran importancia de la minería, por todos los motivos citados anteriormente, cabría pensar que una gran parte de los fondos destinados a la cooperación internacional desde los diferentes países van a parar a esta causa por la gravedad de su situación. Sin embargo, según las estadísticas publicadas por la Organización para la Cooperación y el Desarrollo Económico OECD (2008), sobre la distribución de la ayuda según los principales objetivos de los países que participan en la Asistencia Oficial para el Desarrollo (AOD), España dedica tan sólo el $3.2 \%$ de su ayuda a la cooperación en el bloque de la Industria, Minería y Construcción, en el cual no se distingue el porcentaje en cada uno de los diferentes campos. Cualquier observador de este dato se podría demandar por qué se destina un porcentaje tan bajo a una actividad tan relevante en los países más necesitados. Sin duda alguna, gran parte de la explicación viene dada por la propia actividad con la que se pretende cooperar. De todas las organizaciones que trabajan con la cooperación para el desarrollo, son muy pocas las que se centran en los trabajos relativos a la minería, pues ésta tiene mala fama a nivel mundial. No son pocas las personas que se asombran al escuchar que una ONG se pueda dedicar principalmente a la minería, pues el propio nombre llena de dudas a la población en general y muchas personas no apostarían por una actividad que tiene tan mala reputación. Sin duda, esta apreciación está unida a un desconocimiento total de este campo de la industria, imprescindible en la sociedad en la que vivimos. Aún así no son menos ciertos los datos que indican que a lo largo de la historia han ido de la mano de la minería tradicional graves problemas relacionados con la contaminación y la explotación laboral. Solventar parte de esta problemática es uno de los objetivos de las asociaciones y organizaciones que se dedican al campo de la minería, pero ¿en qué terrenos de la minería se puede cooperar?

Algunos de los campos en los que se centran los trabajos de cooperación de las diferentes agencias y organizaciones son: técnicos, de productividad, sociales y culturales, de género y trabajo infantil, comunitarios, organizativos, ambientales, de salud y seguridad, legales, de formalización, laborales, económicos, de financiamiento y de gestión empresarial (Hruschka, 2002). En Ecuador la incorporación de técnicos en las labores de producción por los diferentes proyectos de asistencia técnica e internacional, generaron los siguientes resultados durante la década de los 90, que afectaron a más de 10,000 pequeños mineros: aplicación de sistemas de explotación con mejoras en la seguridad, incorporación de técnicas en voladuras, incorporación de mejores sistemas de transporte de material en la mina, incorporación parcial de equipos de seguridad, incorporación parcial de retortas para destilación de amalgamas, incorporación de procesos de cianuración, toma de conciencia ambiental e incorporación del 90\% de la actividad minera a pequeña escala en la Legislación Minera de dicho país (Sandoval, 2001).

La gran cantidad de proyectos que se han llevado a cabo en algunos países de Sudamérica, de los cuales se puede ver un ejemplo en la Tabla 6 , realizados desde organizaciones públicas y privadas, dan a pensar que la problemática ambiental y laboral de las regiones mineras artesanales ha mejorado sustancialmente en dichos asentamientos mineros, pero se conoce que no sólo no ha habido una clara mejoría de la situación sino que, al contrario, en algunas zonas se ha empeorado su práctica.

\section{Metodología de un proyecto de cooperación enfocado a la pequeña minería}

Los proyectos de cooperación constan de varias fases (Figura 1) que se irán realizando sucesivamente a medida 
Tabla 6. Algunas actuaciones de cooperación en América Latina de diferentes ONGs y agencias de cooperación.

\begin{tabular}{|c|c|c|}
\hline Nombre & Actuaciones & Tipología ayuda \\
\hline $\begin{array}{l}\text { Centro de estudios so- } \\
\text { ciales y publicaciones }\end{array}$ & $\begin{array}{l}\text { Perú. Plan de Acción del Comité Directivo Nacional para la Preven- } \\
\text { ción y Erradicación del Trabajo Infantil (CPETI) para erradicar el } \\
\text { trabajo infantil en minas artesanales y canteras. }\end{array}$ & Trabajo infantil \\
\hline $\begin{array}{l}\text { Centro Canadiense de } \\
\text { Estudios y Cooperación } \\
\text { Internacional (CECI) }\end{array}$ & $\begin{array}{l}\text { Bolivia. Proyecto Reformin (Formación de Formadores en Negoci- } \\
\text { ación y Transformación de Conflictos en el Sector Minero de Bolivia). } \\
\text { Perú y Bolivia. Mejora de las condiciones de vida y de trabajo de los } \\
\text { niños mineros y sensibilización de los padres sobre el futuro de los } \\
\text { niños. }\end{array}$ & $\begin{array}{l}\text { Pacificación. } \\
\text { Prevención y resolu- } \\
\text { ción de conflictos. } \\
\text { Trabajo infantil }\end{array}$ \\
\hline $\begin{array}{l}\text { Asociación Solidaria } \\
\text { para el Desarrollo }\end{array}$ & $\begin{array}{l}\text { Perú. Programa de Desarrollo Minero Artesanal (PDMA): implemen- } \\
\text { tar una propuesta de desarrollo desde una perspectiva integral conside- } \\
\text { rando estrategias a nivel económico, productivo, social, ambiental, de } \\
\text { salud y educación. }\end{array}$ & $\begin{array}{l}\text { Medio ambiente } \\
\text { (EIA). } \\
\text { Capacitación. } \\
\text { Promoción de mujeres } \\
\text { y niños, trabajo } \\
\text { infantil }\end{array}$ \\
\hline $\begin{array}{l}\text { Asociación de Mineros } \\
\text { del Sur Medio y Centro } \\
\text { del Perú (AMASUC) }\end{array}$ & $\begin{array}{l}\text { Perú. (2005) Taller de capacitación en procedimientos legales y ad- } \\
\text { ministrativos para la minería. }\end{array}$ & $\begin{array}{l}\text { Capacitación en pro- } \\
\text { cedimientos legales }\end{array}$ \\
\hline $\begin{array}{l}\text { Cooperación técnica del } \\
\text { Gobierno Suizo (COTE- } \\
\text { SU) }\end{array}$ & $\begin{array}{l}\text { Ecuador. Proyecto COSUDE: minimización de las emisiones de } \\
\text { mercurio en minas de oro; introducción de tecnologías para mejorar } \\
\text { la explotación y beneficio mineral; capacitación para la producción de } \\
\text { equipos económicos para la pequeña minería; fortalecimiento a las in- } \\
\text { stituciones locales para continuar las acciones en favor del medio am- } \\
\text { biente y asesoramiento técnico a los mineros, sociedades mineras para } \\
\text { la solución de problemas geológico-mineros y medio ambientales. } \\
\text { Proyecto PRODEMINCA: investigaciones metalúrgicas, con la } \\
\text { asistencia técnica del Swedish Environmental Systems (SES) para } \\
\text { mejorar el conocimiento de los diferentes tipos de mineralizaciones } \\
\text { auríferas y sus procesamientos. }\end{array}$ & $\begin{array}{l}\text { Sostenibilidad ambi- } \\
\text { ental. } \\
\text { Capacitación. } \\
\text { Apoderación de las } \\
\text { instituciones locales. } \\
\text { Aportación de cono- } \\
\text { cimientos en geología }\end{array}$ \\
\hline CODIGEM & $\begin{array}{l}\text { Ecuador. Proyectos del gobierno con el apoyo de la Corporación } \\
\text { de Investigación es Geológico Minero Metalúrgicas (COGIDEM) } \\
\text { y la Cámara Nacional de la Pequeña a Minería (CAPEMINE) en la } \\
\text { capacitación de los mineros artesanales. }\end{array}$ & Capacitación \\
\hline $\begin{array}{l}\text { Dirección Nacional de } \\
\text { Geología }\end{array}$ & $\begin{array}{l}\text { Ecuador. Cooperación en la Reforma de la Ley de Minería y del } \\
\text { Reglamento General de la Ley de Minería de Abril de } 2001 .\end{array}$ & Legal \\
\hline OIKOS & Ecuador. Realización de auditorías medioambientales & Ambiental \\
\hline $\begin{array}{l}\text { Instituto Latinoameri- } \\
\text { cano de Investigaciones } \\
\text { Sociales }\end{array}$ & Ecuador. Capacitación en temas socioambientales & Ambiental \\
\hline $\begin{array}{l}\text { Centro de Tecnología } \\
\text { Mineral del Ministerio } \\
\text { de Industria }\end{array}$ & $\begin{array}{l}\text { Brasil. Proyecto sobre el impacto del mercurio en la cuenca del Rio } \\
\text { Tapajos. }\end{array}$ & Ambiental \\
\hline $\begin{array}{l}\text { Empresa Nacional de } \\
\text { Minería }\end{array}$ & $\begin{array}{l}\text { Chile. Programas de acceso a financiamiento y de apoyo geológico en } \\
\text { las labores de reconocimiento de reservas. }\end{array}$ & $\begin{array}{l}\text { Aportación de cono- } \\
\text { cimientos en geología. } \\
\text { Capacitación Finan- } \\
\text { ciera }\end{array}$ \\
\hline
\end{tabular}




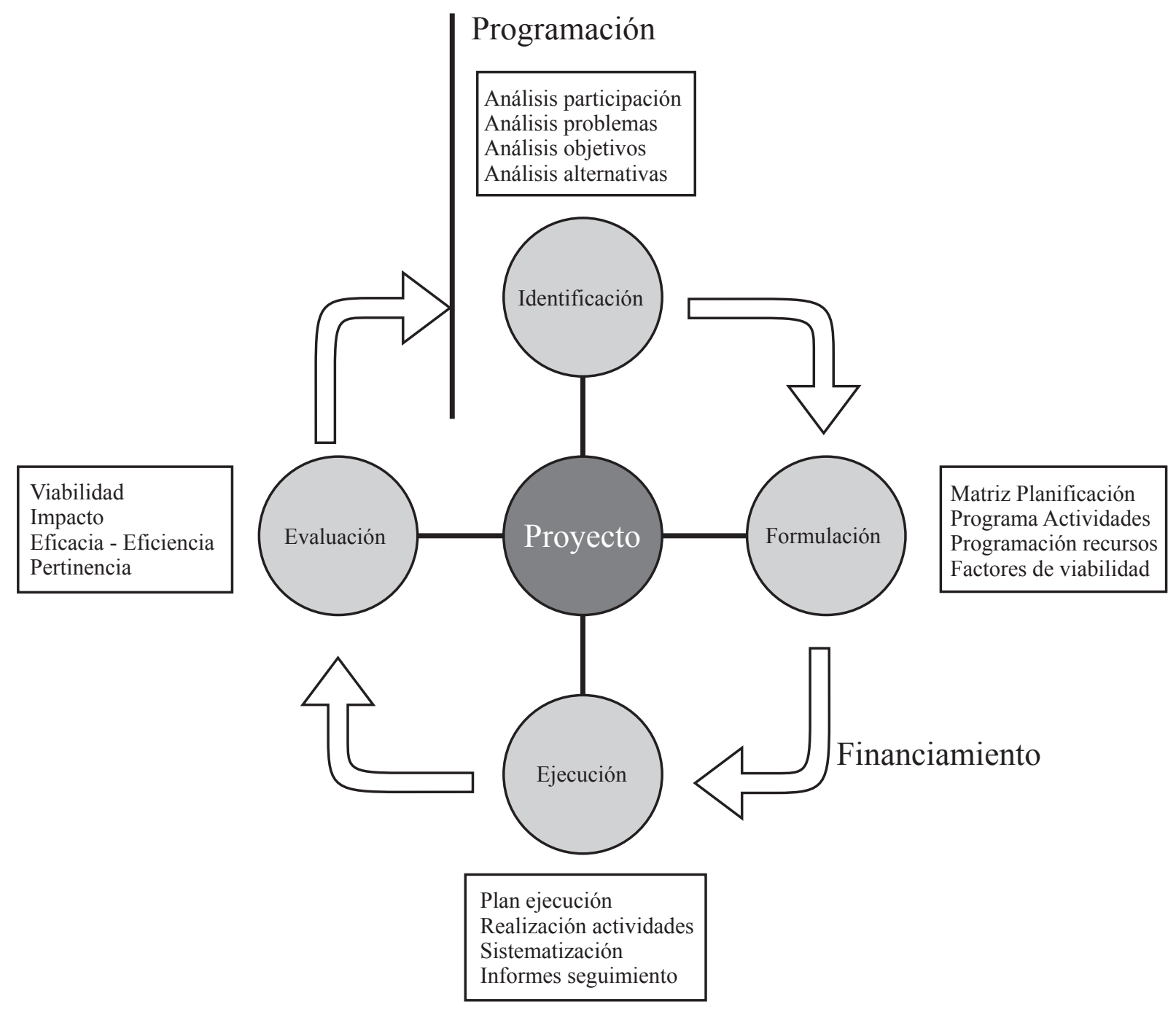

Figura 1. Ciclo de un proyecto de cooperación, modificado de Gómez y Sainz (1999).

que la fase anterior se ha ejecutado de manera óptima (Gómez y Sainz, 1999). Estas fases se engloban dentro de lo que se considera el Enfoque del Marco Lógico, que desde su aparición inicial, hace casi treinta años, ha sido el método más utilizado por la mayor parte de las agencias de cooperación internacional para la planificación y gestión de proyectos de desarrollo (Camacho et al., 2001). El EML es una metodología de planificación de proyectos orientada a la consecución de objetivos y se caracteriza por ser un procedimiento de planificación por pasos sucesivos, por la documentación permanente de los pasos de planificación y por buscar siempre un enfoque desde el consenso.

De las diferentes fases de un proyecto de cooperación, la que decidirá gran parte del éxito del proyecto será la identificación del mismo. La primera pregunta que una ONG debe hacerse es si puede cooperar en determinado proyecto y alcanzar los objetivos propuestos.

Para conocer los marcos de actuación de una ONG, ésta deberá definir un Plan Estratégico planteándose cuáles son sus objetivos para el futuro. En este caso, una ONG en cuyos objetivos esté cooperar con la pequeña minería artesanal debería considerar cuáles son sus ámbitos de actuación en la misma y en qué campos puede cooperar para mejorar la calidad de vida de las personas involucradas.

A menudo la actividad minera está dirigida por grandes compañías que tienen como objetivo prioritario la obtención de los máximos beneficios posibles, que se distribuyen de forma muy desigual remitiendo escasas ganancias a la población local. Cabría pensar que una actividad económica de tal calibre estuviera totalmente regulada y se ejecutara de la manera más salubre para los trabajadores y el entorno, dado que es una actividad global y milenaria, pero por el contrario, la explotación de los recursos geológicos se está desarrollando de una manera desigual y en algunos casos altamente nociva.

Los recursos geológicos que ofrece el planeta han sido 
fuente de desarrollo económico y social de la humanidad hasta el punto que evolución y explotación minera han ido cogidas de la mano. Prácticamente cualquier utensilio de los que se encuentran a nuestro alrededor tiene su origen en un recurso geológico, es por ello que la explotación de los mismos forma parte de una de las industrias consideradas como parte del motor económico mundial.

La demanda planetaria de los recursos geológicos crece de una manera elevada a medida que aumenta la población mundial. Cabe recordar que el consumo de dichas substancias se produce de un modo muy disímil entre los denominados países del norte y los del sur. Así, según Suslick (1992), cada habitante de un país desarrollado consume o es responsable de la manipulación y uso de 20 toneladas de recursos geológicos al año. Este valor llevado a los extremos lo representa Estados Unidos, que requiere para su propio uso el $30 \%$ de todas las materias primas consumidas por la humanidad. La demanda desigual de los recursos geológicos por los diferentes países ha generado una industria de explotación que se desarrolla en los lugares donde aquellos son más abundantes, el control medioambiental es menor y las exigencias en seguridad y salud laboral son pocas o inexistentes. Esta búsqueda del menor coste a toda costa provoca una presión política, económica, social y ambiental muy fuerte en aquellos países en vías de desarrollo, presión que en algunos casos acaba dando pie a un conjunto de conflictos bélicos (Mata, 2006).

\section{Análisis de las Intervenciones}

En la Escola Politècnica Superior d'Enginyeria de Manresa (Cataluña, España) se han creado dos ONGs que desarrollan su actividad, total o parcialmente, en el campo de la minería. Una de ellas se denomina Minería para el desarrollo (en adelante MPD) y la otra es la Associació d'Estudiants $i$ Enginyers Tècnics de Mines, Industrials $i$ Telecos de Manresa Pel Desenvolupament (en adelante AMIT). Esta última, como su nombre indica, además de minería trata de diversos temas relacionados con otras ramas de la ingeniería. Una gran parte de los recursos económicos de estas ONGs provienen de un organismo de cooperación de la Universitat Politècnica de Catalunya denominado Centro de Cooperación para el Desarrollo, CCD, que subvenciona parte del viaje de los cooperantes expatriados.

Todas las actuaciones de estas ONGs, hasta el momento, se han realizado en Latinoamérica: en Nicaragua y Ecuador, Colombia, Perú (Figura 2), además de Angola.

\subsection{Nicaragua}

La AMIT realiza sus proyectos de cooperación principalmente en las tres poblaciones principales del triángulo minero: Siuna, Rosita y Bonanza, localizadas en la Región Autónoma del Atlántico Norte (RAAN). Esta cooperación comenzó en el año 2005.

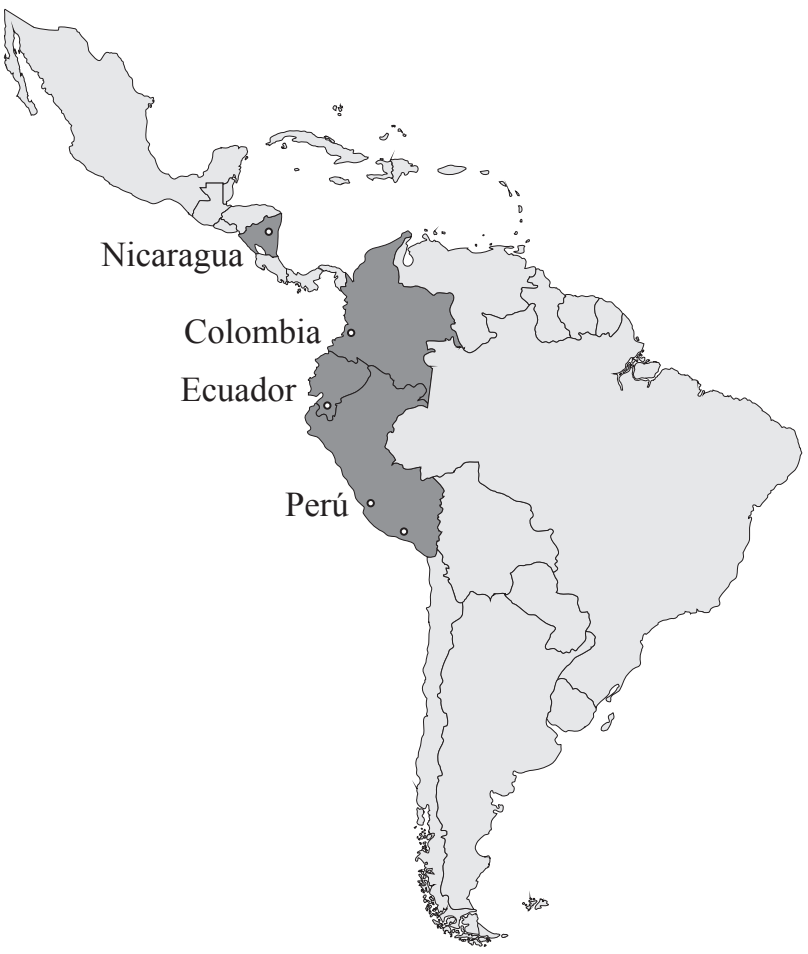

Figura 2. Localización de las diferentes actuaciones de las ONGs MPD y AMIT.

En el triángulo minero la principal y casi única fuente de ingresos es la minería del oro. Además de los empleados de las empresas mineras legales están los denominados "güiriseros" o mineros artesanales. Agrupados por sindicatos, la mayoría extraen el mineral desde sus propias minas artesanales dentro de la concesión minera de una empresa, normalmente en las partes donde la ley del mineral es más baja. Están obligados a vender a la empresa el mineral extraído, del que demandan un diámetro específico, inferior a 3" (pulgadas), y ésta les marca el precio de compra y las entregas de material. La compra se efectúa mediante programaciones de $10 \mathrm{t}$ que tienen que ser entregadas en un momento concreto y ellas se reparten entre las cooperativas que se encargan de distribuirlas entre los mineros. Estas programaciones son repartidas por los sindicatos de forma arbitraria y muchos "güiriseros" siguen trabajando con pocas o sin programaciones esperando la ocasión de poder vender.

Esta actividad de minería artesanal está expuesta a gran número de peligros, sin un conocimiento técnico preciso ni capacidad de mejora. Esto representa un continuo peligro para los mineros artesanales que exponen la vida durante su jornada laboral debido a la precariedad de su actividad. Por ello, las actividades de cooperación en este sector han estado encaminadas principalmente al asesoramiento técnico con el propósito de implementar un sistema de explotación que promueva una minería artesanal más segura. Para ello, se han impartido actividades de formación a los mineros a lo 
largo de todos los proyectos llevados a cabo en este lugar, en las que se realizaron cursos de formación centrados en seguridad minera y explosivos.

Adicionalmente, se ha realizado un inventario de los lugares de interés socio-paisajístico para intentar encontrar una nueva actividad económica que no dependa exclusivamente de la minería y que los ciudadanos del triángulo minero puedan promocionar el eco-turismo.

Tras el primer año, se realizó un segundo proyecto, en 2006, que consistió en la ampliación del inventario de las rutas eco-turísticas. Además, se hizo un estudio de las rastras (máquinas de trituración y molienda) de los mineros artesanales describiendo las problemáticas existentes de funcionamiento y buscando las soluciones idóneas a aplicar.

El tercer proyecto, en el 2007, estuvo enfocado hacia la seguridad de los mineros y donación de equipos de protección individual. Además se realizó un estudio de las aguas de zonas pobladas y mineras de Bonanza que se analizaron para cuantificar la presencia de cianuro.

El cuarto proyecto, en 2008, consistió en la construcción de una polea con seguridad antiácida; la implantación de un sistema de comunicación por radiofrecuencia entre comunidades y los análisis de los posibles focos contaminantes de agua producidos por las escombreras mineras.

Los resultados fueron positivos ya que el reconocimiento de las zonas mineras permitió conocer sus necesidades para poder preparar futuros proyectos y así satisfacer necesidades concretas (Tabla 4).

\subsection{Colombia y Ecuador}

Previamente a constituirse la ONG MPD se iniciaron algunas actividades de cooperación en Colombia y Ecuador. En Colombia se realizó una valoración turística del sistema cárstico de La Danta (Antioquía) para contribuir a la conservación del patrimonio geológico del lugar, además de ofrecer la posibilidad de generar una nueva fuente económica para el futuro.

El proyecto se realizó mediante una campaña de campo que puso de manifiesto el valor de las cavernas Danta, Marleny y Heider como recurso turístico. Con los datos

Tabla 4. Detalle del proyecto de cooperación desarrollado en Nicaragua.

\section{Título}

Participantes y titulación

Contraparte

Localización de la acción

Problemática

Objetivos del proyecto

Actividades realizadas

Valoración

Proyectos posteriores en el área

Proyectos posteriores en el área

\section{Proyecto de Cooperación en el Triángulo Minero, Nicaragua. Fases 1, 2 y 3}

Estudiantes de ingeniería técnica de minas e ingeniería mecánica de de la ONG AMIT.

Central Sandinista de Trabajadores (CST) de Nicaragua.

Triángulo minero: poblaciones de Siuna, Rosita y Bonanza, Región Autónoma del Atlántico Norte (RAAN).

- Minería insegura.

- Condiciones de mercado poco ventajosas y sectarias.

Asesoramiento técnico para la implementar el sistema de explotación de la minería artesanal del triángulo minero.

- Impartir actividades de formación a los mineros

- Inventariar los lugares de interés socio-paisajístico para intentar encontrar una nueva actividad económica que no dependa exclusivamente de la minería y que los ciudadanos del triángulo minero puedan promocionar el eco-turismo.

La valoración final fue positiva, ya que el reconocimiento de las zonas mineras permitió conocer sus necesidades para poder preparar futuros proyectos para satisfacer necesidades concretas.

Segundo proyecto (2006):

- Ampliación del inventario de las rutas eco-turísticas.

- Estudio de la problemática de las rastras de los mineros artesanales y propuesta de soluciones a aplicar.

Tercer proyecto (2007):

- $\quad$ Seguridad de los mineros, donación de equipos de protección individual.

- Análisis de las aguas de Bonanza para cuantificar la presencia de cianuro.

Cuarto proyecto (2008):

- Construcción de una polea con seguridad antiácida.

- Implantación de un sistema de comunicación por radiofrecuencia entre comunidades.

- Análisis de posibles focos contaminantes de agua producidos por las escombreras mineras.

En todos ellos se realizaron cursos de formación a mineros en seguridad minera y explosivos. 
obtenidos se elaboró una base de datos para iniciar un catastro de cuevas en la región. Para valorar las cavernas se crearon tablas considerando los siguientes factores: recurso turístico, conservación/degradación y seguridad/riesgos. A partir de los datos obtenidos se efectuaron recomendaciones para la protección y buen uso de las mismas. Al mismo tiempo se realizaron campañas de concienciación de los recursos naturales ofreciendo una nueva perspectiva de uso para los vecinos del corregimiento de La Danta, en las que se promovió la divulgación mediante la realización de paneles $\mathrm{y}$ fichas informativas que se cedieron al corregimiento. El proyecto se realizó según lo previsto pero se desconoce si se aplicaron las propuestas por parte del municipio (Tabla 1).

En el caso de Ecuador (Tabla 2) se realizó un plan de mitigación de los riesgos geodinámicos en el sector de Zaruma, situado en la provincia de El Oro. La ciudad se asienta en el flanco oeste de la Cordillera Occidental de Los Andes.

El oro y la plata han sido explotados durante muchos siglos en las montañas de Zaruma, pues ya los incas producían oro en la zona cuando los españoles llegaron en el año 1549. La extracción de oro se aceleró al entrar la empresa norte-americana Southern American Development Company (SADCO), que obtuvo el control de los principales depósitos de oro del distrito en el año 1896. En 1984 los pozos de la SADCO fueron irrumpidos por mineros que vivían en la miseria y se comenzó a desarrollar una minería de tipo artesanal y de pequeña escala. Las minas artesanales son inseguras, están mal ventiladas y, al no estar hechas a partir de ningún tipo de estudio, sufren hundimientos constantes.
La minería informal es una actividad de riesgo para los propios trabajadores, el entorno e infraestructuras cercanas a las galerías, que se pueden ver afectadas por los hundimientos o por las vibraciones de las explosiones en éstas. El laboreo se efectúa mayormente en galerías abandonadas por antiguas explotaciones formales, a menudo concentrándose en los pilares que sostienen las cámaras, la desaparición de los cuales puede provocar el colapso de las galerías.

El objetivo del proyecto era estudiar la problemática de una zona gravemente afectada por las inestabilidades del terreno, determinar las causas que la generan, establecer en qué grado interviene la minería y proponer una serie de recomendaciones para minimizar los efectos que estas inestabilidades generan en las diferentes infraestructuras del municipio.

Gracias a los resultados obtenidos a partir del trabajo de campo y la previa documentación bibliográfica y la información conseguida a partir de entrevistas a técnicos y habitantes del municipio, se desarrolló un informe que se cedió a la municipalidad dando información sobre las zonas de mayor riesgo del municipio. Un año más tarde uno de esos sectores se vio gravemente afectado por una avenida de lodo y agua durante la época de lluvias. Aún así se desconoce si la municipalidad ha tomado mayores medidas al respecto.

\subsection{Perú}

La primera actividad de la ONG Minería Para el Desarrollo fue el proyecto de desarrollo de las potencialidades de la

Tabla 1. Detalle del proyecto de cooperación desarrollado en Colombia.

\begin{tabular}{|c|c|}
\hline Título & Valoración turística del sistema cárstico de La Danta (Antioquia) \\
\hline Participantes & 1 Estudiante de ingeniería técnica de minas y geóloga, 1 Ingeniera geóloga, 1 Profesor \\
\hline Contraparte & Sociedad Espeleológica de Colombia y corregimiento de La Danta. \\
\hline Localización de la acción & Corregimiento de La Danta, municipio de Sonsón, Antioquia, en el Magdalena Medio. \\
\hline Problemática & $\begin{array}{l}\text { La riqueza geológica y ecológica de esta zona es amenazada por la minería artesanal del mármol y } \\
\text { la visita destructiva de algunas personas. }\end{array}$ \\
\hline Objetivos del proyecto & $\begin{array}{l}\text { Valoración turística de las cavernas de La Danta para clasificar las explotaciones mineras artesana- } \\
\text { les como patrimonio geológico del lugar. }\end{array}$ \\
\hline Actividades realizadas & $\begin{array}{l}\text { - Campaña de campo y elaboración de la descripción espeleológica y la valoración como } \\
\text { - } \quad \text { Realización de una base de datos para iniciar un catastro de cuevas en la región. } \\
\text { - Recomendaciones para la protección y buen uso de las cavernas. } \\
\text { - Campañas de concienciación de los recursos naturales ofreciendo una nueva perspectiva de } \\
\text { uso para los vecinos del corregimiento de La Danta. Divulgación mediante paneles y fichas } \\
\text { informativas. }\end{array}$ \\
\hline Valoración & $\begin{array}{l}\text { El proyecto se realizó según lo previsto. Sin embargo se desconoce si se aplicaron las propuestas } \\
\text { por parte del municipio. }\end{array}$ \\
\hline
\end{tabular}


Tabla 2. Detalle del proyecto de cooperación desarrollado en Ecuador.

\begin{tabular}{|c|c|}
\hline Título & Plan de mitigación de los riesgos geodinámicos en Zaruma \\
\hline Participantes & 2 Estudiantes de ingeniería técnica de minas y geólogas, 1 Ingeniera geóloga, 1 Profesor \\
\hline Contraparte & Escuela Superior Politécnica del Litoral-ESPOL y empresa BIRA, S.A. \\
\hline Localización de la acción & Zaruma, Provincia de El Oro. \\
\hline Problemática & $\begin{array}{l}\text { Explotaciones artesanales de oro: inseguridad en las minas y mala ventilación. El laboreo se } \\
\text { efectúa mayormente en galerías abandonadas por antiguas explotaciones formales, a menudo } \\
\text { concentrándose en los pilares que sostienen las cámaras. }\end{array}$ \\
\hline Objetivos del proyecto & $\begin{array}{l}\text { Estudiar la problemática de una zona gravemente afectada por las inestabilidades del terreno, } \\
\text { determinar las causas que las generan, establecer en qué grado interviene la minería y proponer } \\
\text { una serie de recomendaciones para minimizar los efectos que estas inestabilidades generan en las } \\
\text { diferentes infraestructuras del municipio. }\end{array}$ \\
\hline Actividades realizadas & $\begin{array}{l}\text { - Estudio bibliográfico en Zaruma, y entrevistas con técnicos y habitantes del municipio. } \\
\text { Trabajo de campo en sectores con un elevado riesgo para la integridad de los habitantes y/o } \\
\text { con patologías importantes. } \\
\text { Elaboración de un informe de cada punto con indicación de las causas y medidas adoptadas } \\
\text { actualmente y las medidas recomendadas. }\end{array}$ \\
\hline Valoración & $\begin{array}{l}\text { El trabajo se desarrolló y se entregó un informe a la municipalidad con los resultados obtenidos, } \\
\text { pero se desconoce si se han tomado medidas al respecto. Un año después de la realización del } \\
\text { proyecto una de las zonas determinadas de alto riesgo se vio seriamente afectada por un mov- } \\
\text { imiento de tierras durante la época de lluvias. }\end{array}$ \\
\hline
\end{tabular}

minería en algunas comunidades mineras de Huancavelica, Perú (Tabla 3). Algunos yacimientos y antiguas minas abandonadas de este departamento son explotados por individuos o grupos familiares utilizando técnicas y equipos muy rudimentarios, arriesgando su vida día a día pues no poseen conocimientos sobre las técnicas mineras. A esto se une una inadecuada comercialización de los productos obtenidos, los cuales normalmente son comprados a un precio muy por debajo de su valor real. Las actividades realizadas para contribuir a aminorar esta problemática fueron las siguientes:

a) en la zona de Julcani se realizó una identificación de los riesgos laborales del interior de la mina y de las instalaciones anexas; la elaboración de un plan de mejora y la realización de un curso de capacitación para identificar riesgos y prevenirlos; se analizó además el método utilizado para neutralizar la aguas ácidas como residuo de la actividad minera.

b) en la zona de Santa Bárbara se realizó un análisis in situ de la situación actual de las minas; la elaboración de un listado con todos los posibles elementos del patrimonio minero de la zona; la visita en detalle de todos los puntos de interés seleccionados, rellenando para cada uno una ficha de campo con los datos sobre la situación, descripción, croquis o fotografías, tipo de interés y el tipo y grado de vulnerabilidad; ampliación de la lista con otros puntos de interés encontrados sobre el terreno; elaboración de una propuesta de varios circuitos turísticos de la Ruta del
Mercurio.

Actualmente se está realizando un Proyecto de cooperación minero-ambiental para la mejora de la calidad de vida de los habitantes del asentamiento minero artesanal de Misky, Arequipa. Las actuaciones de intervención tienen por objeto reducir la incidencia de los problemas medio ambientales relacionados con actividades mineras y establecer las bases para una minería segura.

La valoración general del proyecto es positiva a pesar de que no se han conseguido los objetivos planteados por el escaso conocimiento de la contraparte, pues no trabajaban con la minería artesanal como en un inicio se había creído y porque el estudio del patrimonio minero de las minas de Santa Bárbara estaba mucho más desarrollado de lo que la contraparte informó previamente. Aún así, aunque la aportación de nuestra cooperación en ese proyecto no fue plenamente satisfactoria, se considera que la experiencia fue positiva ya que se ha contactado con contrapartes que sí trabajan directamente con la minería artesanal del país y se ha podido realizar un reconocimiento del terreno, muy útil para futuros proyectos.

\section{Análisis de los resultados de la cooperación}

\subsection{Proyectos de MPD y AMIT}

Los proyectos llevados a cabo por MPD y AMIT, a 
pesar de representar un gran esfuerzo y dedicación por parte de los cooperantes, no han tenido un éxito completo, pues en muchos casos la falta de seguimiento hace que se desconozca el alcance de los mismos, como en el caso de La Danta en Colombia o Zaruma en Ecuador (Tablas 1 y 2). Se podría decir que estos proyectos no han sido plenamente exitosos pues la falta de medios y el hecho de no estar creados dentro de un programa de desarrollo local o regional mucho más amplio hace que se proyecten desde países lejanos al lugar donde se realizan. Es imposible que desde el país de origen de las ONGs de la cooperación internacional se pueda diseñar un proyecto viable en una región del sur, pues se hace con desconocimiento del contexto, de las técnicas apropiadas y de la cultura del país con el que se pretende cooperar. Los conocimientos técnicos de estudiantes y profesionales no son suficientes para enraizar una propuesta en una comunidad y mucho menos si el trabajo de campo y el contacto con dicha comunidad se lleva a cabo en apenas unos meses. La vinculación con la contraparte y el trabajo conjunto con los actores del sur son lo que se podría denominar un buen comienzo. En el caso de no existir una apropiada comunicación con la contraparte se puede llegar a diseñar proyectos alejados de la realidad del contexto donde se va a trabajar como sucedió en Julcani y las Minas de Santa Bárbara en Perú (Tabla 3),

Por otro lado, la falta de trabajo previo con la comunidad para determinar los problemas reales que padecen provoca que las dificultades que se observan en una región no sean las que la población considere como prioritarias y, en ocasiones, estos problemas, que deberían generar el objetivo especifico de todo proyecto de cooperación, se confunden con un sinfín de actividades que, aunque bien planteadas, no ayudan a mejorar la calidad de vida de los beneficiarios. Sin embargo, ¿cómo podemos determinar en qué grado estos

Tabla 3. Detalle del proyecto de cooperación desarrollado en Perú.

\section{Título}

Participantes

Contraparte

Localización de la acción

Problemática

Objetivos del proyecto

Actividades realizadas

Valoración

\section{Desarrollo de las potencialidades de la minería en las comunidades mineras de Huancavelica}

4 estudiantes de ingeniería de minas e ingeniería química, 1 geólogo, 1 químico y 2 profesores

Asociación Civil de San Javier de Perú

Departamento de Huancavelica, al suroeste del Perú.

Explotaciones mineras por individuos o grupos familiares con técnicas y equipos rudimentarios, sin condiciones de seguridad.

Inadecuada comercialización de los productos.

1. Elaboración de un plan de mejora en las labores mineras, en la pequeña minería artesanal.

2. Elaboración de una propuesta de planes de sensibilización social de la unidad minera de Huancavelica y de las comunidades afectadas.

3. Identificación y evaluación del potencial del patrimonio minero.

4. Propuesta de gestión sostenible de la futura declaración de las minas de Santa Bárbara como Patrimonio Cultural de la Humanidad.

Zona de Julcani:

- Identificación de riesgos laborales y elaboración de un plan de mejora.

- Realización de un curso de capacitación para identificar y prevenir los riesgos laborales.

Zona de Santa Bárbara:

- Análisis in situ de la situación actual de las minas.

- Elaboración de un listado de lugares patrimonio minero de la zona.

- Elaboración para cada uno de una ficha de campo con la situación, descripción, croquis, fotografías, tipo de interés y grado de vulnerabilidad.

- Elaboración para cada uno de una ficha de campo con la situación, descripción, croquis, fotografías, tipo de interés y grado de vulnerabilidad.

- $\quad$ Elaboración de una propuesta de varios circuitos turísticos de la Ruta del Mercurio.

La valoración general del proyecto es positiva a pesar de que no se han conseguido los objetivos planteados por:

1. Desconocimiento de la contraparte; inicialmente se creía que la contraparte estaba trabajando con la pequeña minería artesanal, pero resultó que con quien realmente colaboraban ellos era con una gran empresa minera.

2. El estudio del patrimonio minero de las minas de Santa Bárbara previamente estaba mucho más elaborado y avanzado de lo que la contraparte informó previamente.

Por todo esto, se valora que la aportación no ha sido efectiva. 
proyectos han beneficiado a un conjunto de la población sobre la que se ha actuado? Sin duda, ésta es otra de las incorrecciones que encontramos en todos estos proyectos: la falta de marcadores de evaluación en cada uno de los pasos del proceso y en el conjunto del mismo. Desde luego, es indispensable que se establezcan indicadores antes de comenzar el trabajo que nos revelen si se está planificando y realizando mejor o peor el trabajo de cooperación. Por último, si se ha definido el objetivo específico de una manera adecuada, el nivel de consecución del mismo nos orientará sobre el grado del éxito del proyecto.

Asimismo, no se debe considerar que estas actuaciones, por no ser exitosas desde el punto de vista más purista de la cooperación, sean vacuas, pues permiten que muchas personas tengan su primer contacto con las acciones de cooperación, puedan transmitir sus conocimientos y se genere un intercambio cultural entre todas las partes nada menospreciable. Estas pequeñas acciones son las que han dado lugar a la creación de diferentes ONGs técnicas que se abren paso en el mundo de la cooperación con proyectos mucho más ambiciosos y óptimos.

Como ejemplo cabe mencionar los dos proyectos de cooperación que se están gestando actualmente en el seno de la ONG Minería Para El Desarrollo. Uno de ellos se está desarrollando en el poblado minero Misky, en Arequipa, Perú, para el que se han realizado algunas estancias destinadas al reconocimiento a fondo de las comunidades con las que se desea trabajar en proyectos de larga duración. Este proyecto se creó a partir de una propuesta definida por una contraparte conocedora de la problemática del lugar durante largo tiempo. Cabe decir que en el poco tiempo que se viene desarrollando el proyecto de cooperación en Misky los resultados obtenidos son muy positivos pues se han podido desarrollar talleres de formación y se prevén actuaciones futuras que ayuden a mejorar la calidad de vida de los mineros que allí habitan.

Por otro lado, con todo el conocimiento previo adquirido, se ha iniciado un proyecto en Angola con el fin de crear una campaña de colaboración inter-universitaria entre la UPC y la Universidade Agostinho Neto, con el que se contribuirá a la formación de investigadores angoleños mediante un proyecto práctico dirigido a desarrollar la actividad minera a partir de un convenio entre ambas universidades.

\subsection{Posibles efectos negativos de la cooperación}

La cooperación nacional e internacional, tanto de las diferentes agencias como de las ONGs, no siempre consigue sus objetivos, sino que en ocasiones los proyectos, que tardan en desarrollarse varios años y cuestan miles de euros, son infructuosos. Esta esterilidad en los trabajos de cooperación se puede deber a muchos factores, entre ellos a:

- No contar con la contraparte adecuada: en el caso del proyecto desarrollado por la ONG MPD en Huancavelica, Perú, la contraparte no demostró el interés por el proyecto que se esperaba por lo que, después de realizar una primera fase de reconocimiento del proyecto, se decidió no continuar con el programa de actuaciones de mejora de la minería artesanal.

- Realizar proyectos que la población no considera prioritarios para la mejora de su situación actual: en el caso del proyecto de Antioquia, Colombia, realizado por la Universidad Politécnica de Cataluña, se realizó un proyecto con la intención de salvaguardar el patrimonio geológico de una región de Río Claro, que en un futuro podría dotar a la población del lugar de un recurso turístico. En sucesivas reuniones con la población se trató de hacerles ver la importancia de mover las explotaciones de mármol unos centenares de metros para no dañar un conjunto de cavernas de interés espeleológico, incluso se les indicó las claves para su conservación y futura explotación. El hecho de que el proyecto fuera concebido desde Medellín y Manresa (España) y no fuera una demanda de la población de la zona dificultó que estos lo aceptaran como suyo y, por tanto, que se involucraran en él, es por ello que tras la partida de los cooperantes se cree que los trabajos de explotación han continuado donde estaban.

- Escasa permanencia del proyecto: los trabajos de cooperación que se desarrollan en las diferentes regiones requieren de un proceso de consenso y maduración por parte de todos sus participantes y esto demanda indefectiblemente tiempo y recursos. En el caso de las jóvenes ONGs de la Politécnica de Manresa, entre otras muchas, las escasas subvenciones que reciben apenas alcanzan para el viaje de los participantes expatriados durante las vacaciones de éstos. Esto provoca que el trabajo desarrollado en la zona de actuación sea intenso pero breve, por lo que durante la estancia de los cooperantes se realiza un valioso y duro trabajo pero cuando éstos marchan el proyecto se paraliza, pues no cuenta con la continuación de otros cooperantes locales o internacionales. Este hecho ocasiona que sólo se puedan llevar a cabo proyectos de cooperación que se ejecutan en un año y no en programas más extensos, que son los que pueden calar hondo allí donde se llevan a cabo. En los proyectos expuestos de Antioquia, Colombia, y Huancavelica, Perú, el trabajo se desarrolló en una sola fase $\mathrm{y}$, por tanto, en un único año. Por el contrario, los proyectos de Nicaragua y Perú se han desarrollado estacionalmente en varias fases, hecho que ha permitido observar la continuidad del mismo y mantener el contacto con la contraparte, además de desarrollar una mayor cantidad de actuaciones.

- No contar con el apoyo de los grupos de poder y/o gobierno: no son escasos los proyectos que se realizan desde las ONGs en los que se trabaja con una contraparte beneficiaria sin contar con el apoyo de los órganos de poder. En muchos casos la propia situación del lugar hace que no se puedan desarrollar proyectos de cooperación por el clima contrario que se puede generar a las actuaciones de la misma. Es evidente pensar que una acción de cooperación no será acogida del mismo modo por todos los habitantes de una región, pues habrá personas o grupos a los que la situación de desigualdad que existe en una 
comunidad determinada les vaya bien para sus propios intereses; si estos grupos, contrarios a la realización del proyecto de cooperación, son grupos de poder cualquier búsqueda de los objetivos será infructuosa y por tanto toda inversión de tiempo, recursos humanos y dinero vacua. En el caso del proyecto de Río Claro se tuvieron que hacer varias reuniones con representantes del poblado de $\mathrm{La}$ Danta para poder desarrollar los diferentes trabajos de investigación en las cavernas y para poder hacer los talleres de concienciación con la población. Según Sandoval (2001), en el proyecto MINPALCA y Mineros de Ponce Enríquez y Gabi desarrollado en 1997, las negociaciones entre la empresa minera y la minería de pequeña escala se hizo sin la participación de las autoridades gubernamentales no llegando a acuerdos empresariales que posibilitasen el desarrollo minero futuro por una falta de capacidad de negociación por parte de todos los involucrados. En los proyectos de cooperación es imposible alcanzar los objetivos propuestos si no se cuenta con el apoyo de los grupos de poder, es por ello imprescindible establecer una estrategia que vincule a estos grupos con el proyecto a desarrollar.

Por otro lado, la cooperación no siempre tiene los efectos positivos que se desea, sino que puede ser el origen de un conjunto de conflictos que anteriormente al proyecto no existían:

- Generación de conflictos entre diferentes organizaciones: en el proyecto realizado entre Mining Andos, S.A. y Mineros de Nambija, en el 2000, se sobredimensionó la evaluación de las labores mineras que habían realizado en el pasado los pequeños mineros y en consecuencia se realizó una negociación sobre bases no reales que dificultó las relaciones contractuales entre la compañía y los pequeños mineros hasta el punto que, en Septiembre del 2001, el gobierno, a través del Ministerio de Energía y Minas y de las Fuerzas Armadas, tuvo que intervenir para evitar mayores consecuencias (Sandoval, 2001).

- Creación de desigualdades y conflictos entre comunidades: no se debe olvidar que el desarrollo de un proyecto de cooperación en una localidad tendrá como beneficiarios directos e indirectos a grupos de personas concretos que no son siempre los más vulnerables, sino aquellos que se encuentran en un ámbito que permite el desarrollo de un proyecto de cooperación en un momento dado. Cabe recordar que es la propia contraparte la que debe manifestar las necesidades en un determinado tema y, por tanto, la que definirá en gran parte el eje de actuación de un proyecto, al solicitar la cooperación de una ONG determinada. Esta manera de proceder puede suponer que una comunidad representada por una asociación, cooperativa, etc., que sepa moverse en el ámbito de la cooperación internacional se vea más beneficiada que una comunidad con las mismas carencias o más que tenga representantes con menos recursos. Por tanto, es posible ver comunidades donde se están efectuando varios trabajos de cooperación cercanas a comunidades con las mismas carencias que pasan inadvertidas a la ayuda internacional. En este caso es importante dar a conocer a las comunidades circundantes que en un futuro se desarrollarán los mismos proyectos de cooperación en las mismas, siempre cuando esto sea posible.

Por otro lado, en proyectos de cooperación donde se actúa para potenciar el desarrollo económico de un lugar mejorando la infraestructura y la productividad, se está potenciando a un grupo que será más eficaz que los que le circundan. Este contexto crea una situación de agravio comparativo que puede dar lugar a conflictos que anteriormente al desarrollo de un proyecto de cooperación no existían.

\section{Conclusiones}

La práctica de la realización de los proyectos de cooperación en el campo de la minería artesanal en las diferentes geografías ha dejado ver que los ámbitos de actuación de las ONGs que trabajan con la pequeña minería vienen dados por el ciclo mismo de la explotación minera: por la importancia de la geología y minería durante las fases de exploración e investigación, así como para el cálculo de reservas según la ley de mercado y la ingeniería de minas para el óptimo diseño de la explotación, que minimice riesgos, maximice ganancias y sea más tolerable con el entorno. Estos proyectos se generan en el marco de programas de desarrollo social-organizativo, legal, técnico ambiental y empresarial y para que se realicen con éxito deben crear mecanismos que permitan a la contraparte local expresar sus necesidades, crear condiciones que permitan salir a esta minería de la informalidad, abrir el camino para un crecimiento económico del sector e introducir correctas técnicas ambientales tolerantes con el entorno (Hruschka, 2002).

Un modelo a seguir lo constituye la ONG alemana Gesellschaft für Techniche Zusammenarbeit (GTZ), la cual coopera activamente con la pequeña minería de Ghana y ha conseguido que en este país el gobierno de soporte técnico y financiero a la pequeña minería (Hilson, 2002a, b).

Por todo lo comentado previamente se deduce que un proyecto de cooperación con la pequeña minería debería tener presentes factores como la contextualización en un entorno que posee unas características específicas, la vinculación con la contraparte adecuada y programas de desarrollo locales o regionales, la participación de representantes de todos los integrantes de las comunidades en las que se actúa, los aspectos ambientales, la adaptación de las técnicas y tecnologías más apropiadas en el entorno en el que se trabaja, la financiación del programa de cooperación, el compromiso político y, ante todo, tener presente que se pretende acompañar a los beneficiarios en un proceso que deben querer desarrollar ellos mismos. $\mathrm{Y}$ aunque en algunos casos los resultados no sean los 
esperados, en la mayoría de ellos estos proyectos son el único vehículo hacia la tecnificación y regulación de la minería artesanal.

\section{Agradecimientos}

Los autores agradecen al Centre de Cooperació al Desenvolupament de la UPC el financiamiento parcial de los proyectos aquí expuestos en el presente artículo. También agradecemos a los miembros de MPD y AMIT, especialmente a J.M. Mata, J. Font, su valiosa colaboración.

\section{Referencias}

Butt, N.A., 1981, Environment, mining and the Lesser Developed Countries: Environmental Geochemistry and Health, 3, 93-101.

Camacho, H., Cámara, L., Cascante, R., Sainz, H., 2001, El Enfoque del marco lógico: 10 casos prácticos. Cuaderno para la identificación y diseño de proyectos de desarrollo: Madrid, Acciones de Desarrollo y Cooperación A.D.C. Fundación CIDEAL, 235 p.

Instituto para el desarrollo sustentable (EKAMOLLE), 2002, Proyecto de investigación en red sobre organización e institucionalidad en la minería artesanal y en pequeña escala: Lima, Perú, Informe del taller nacional de trabajo UNESCO-IIPM IDRC, $18 \mathrm{p}$.

Gómez, M., Sainz, H., 1999, El ciclo del proyecto de cooperación al desarrollo: La aplicación al marco lógico: Madrid, Fundación CIDEAL, $220 \mathrm{p}$.

Hilson, G., 2002a, Promoting sustainable development in Ghanaian smallscale gold mining operations: The Environmentalist, 22, 51-57.

Hilson, G., 2002b, Technology, managerial and policy initiatives for improving environmental performance in small-scale gold mining industry: Environmental Management, 30, 764-777.

Hruschka, F., 2002, Minería en pequeña escala en Perú. "Perspectiva de la cooperación internacional": Agencia Suiza Para el Desarrollo y la Cooperación (COSUDE), Reporte Técnico, 32 p.
Mata, R., 2006, Pobres per Desastres, Conflictes per Recursos, I Curs de Geologia Per al Desenvolupament: Terrassa, España, Terrassa Solidària, Reporte Técnico, 26 p.

Noetstaller, R., 1994, Small-scale mining, practices, policies, perspectives, in Ghose, A.K. (ed.), Smallscale Mining - A Global Overview: New Delhi, India, Oxford \& IBH, 3-10.

Organisation for Economic Co-operation and Development (OECD), 2008, Development Co-operation Report 2007, Stadistical Annex: Paris, Reporte Técnico Volumen 9, 242 p.

Organización Internacional del Trabajo (OIT), 1999, Social and labour issues in small-scale mines; report for the tripartite meeting on social and labour issues in small-scale mines: Geneva, Suiza, International Labour Office, Reporte Técnico, 99 p.

Organización de las Naciones Unidas (ONU), 2000, Declaración del Milenio: New York, ONU, Resolución aprobada por la Asamblea General, $10 \mathrm{p}$.

Organización de las Naciones Unidas (ONU), 2008, Objetivos de desarrollo del milenio Informe 2008: Nueva York, ONU, Reporte Técnico, 56 p.

Sandoval, F., 2001, La Pequeña Minería en el Ecuador: Inglaterra, Mining, Minerals and Sustainable Development-Internacional Institute for Environment and Development (IIED)-World Business Council for Sustainable Development, $31 \mathrm{p}$.

Spiegel, S.J., Veiga, M.M., 2005, Building Capacity in Small-Scale Mining Communities: Health, Ecosystem Sustainability, and the Global Mercury Project: Eco Health 2, 361-369.

Suslick, S., 1992, Geociências: Um Ensaio Preliminar de Avaliação e Perspectiva: Revista do Instituto de Geociências, 13, 69 - 81.

United Nations Development Programme (UNPD), 2007, Draft regional programme document for Latin America and the Caribbean: New York, UNPD-UN, Reporte Técnico, 13 p.

Veiga, M.M., Maxson, P.A., Hylander, L.D., 2006, Origin and consumption of mercury in small-scale gold mining: Journal of Cleaner Production, 14, 436-447.

Manuscrito recibido: Mayo 17, 2009.

Manuscrito corregido recibido: Febrero 18, 2010.

Manuscrito aceptado: Febrero 25, 2010. 